\title{
LOWER BOUNDS FOR SOLUTIONS OF HYPERBOLIC INEQUALITIES
}

\author{
HAJIMU OGAWA
}

1. Introduction. Let $D$ denote a bounded domain in $E^{n}$ and $I$ the interval $1 \leqq t<\infty$. Let $L$ be the second-order hyperbolic operator

$$
L=\frac{\partial^{2}}{\partial t^{2}}-\sum_{i=1}^{n} \frac{\partial}{\partial x_{i}}\left(a_{i j} \frac{\partial}{\partial x_{j}}\right)
$$

defined on $R=D \times I$. Introducing the norms

$$
\begin{aligned}
\|u(t)\|_{0}^{2} & =\int_{D} u^{2} d x, \\
\|u(t)\|_{1}^{2} & =\int_{D}\left[\left(\frac{\partial u}{\partial t}\right)^{2}+\sum_{i=1}^{n}\left(\frac{\partial u}{\partial x_{i}}\right)^{2}\right] d x,
\end{aligned}
$$

for functions $u$ in $C^{2}(R)$, Protter [4] investigated the asymptotic behavior of solutions of inequalities of the form

$$
\|L u(t)\|_{0} \leqq \phi(t)\|u(t)\|_{1} \text {. }
$$

If $\Gamma$ is the boundary of $D$, he found that any solution of (1.2) which satisfies the conditions

$$
\begin{aligned}
u=0 & \text { on } \Gamma \times I, \\
\lim _{t \rightarrow \infty} t^{\alpha}\|u(t)\|_{1}=0 & \text { for all } \alpha>0,
\end{aligned}
$$

must vanish identically, provided that

$$
\phi(t)=O\left(t^{-1}\right), \quad \frac{\partial a_{i j}}{\partial t}=O\left(t^{-1}\right) .
$$

Conditions for other types of asymptotic behavior have also been studied by Protter [5].

It is the purpose of this paper to find sufficient conditions for the existence of lower bounds of the form

$$
\|u(t)\|_{1} \geqq C\left\|u\left(t_{0}\right)\right\|_{1}[K(t)]^{-1}, \quad t \geqq t_{0} \geqq 1,
$$

where $C$ is a positive constant and $K$ is a differentiable function satisfying

Received by the editors July 22, 1964. 


$$
K(t)>0, \quad K^{\prime}(t) \geqq 0, \quad \lim _{t \rightarrow \infty} K(t)=\infty .
$$

In particular, it will be shown that in the case $K(t)=t^{\alpha}$, a lower bound exists under conditions somewhat weaker than (1.3). The results will also be extended to symmetric hyperbolic operators.

The author wishes to thank M. H. Protter for a number of valuable suggestions.

2. Second-order hyperbolic inequalities. Let $L$ be the operator defined by (1.1). We assume $a_{i j}=a_{j i} \in C^{1}(R)$, and suppose that there are positive constants $m$ and $M$ such that

$$
m \sum_{i=1}^{n} \xi_{i}^{2} \leqq \sum_{i, j=1}^{n} a_{i j} \xi_{i} \xi_{j} \leqq M \sum_{i=1}^{n} \xi_{i}^{2} .
$$

For functions $u \in C^{2}(R)$ we introduce the norm

$$
\|u(t)\|^{2}=\int_{D}\left[\left(\frac{\partial u}{\partial t}\right)^{2}+\sum_{i, j=1}^{n} a_{i j} \frac{\partial u}{\partial x_{i}} \frac{\partial u}{\partial x_{j}}\right] d x,
$$

which is equivalent to the norm $\|u(t)\|_{1}$. If $u=0$ on $\Gamma \times I$, it is easily seen that

$$
\frac{d}{d t}\|u(t)\|^{2}=2 \int_{D} \frac{\partial u}{\partial t} L u d x+\int_{D} \sum_{i, j=1}^{n} \frac{\partial a_{i j}}{\partial t} \frac{\partial u}{\partial x_{i}} \frac{\partial u}{\partial x_{j}} d x .
$$

Hence for any function $K$ satisfying (1.4), we have the identity

$$
\begin{aligned}
\frac{d}{d t}\left[K^{2}(t)\|u(t)\|^{2}\right]= & 2 K(t) K^{\prime}(t)\|u(t)\|^{2}+2 K^{2}(t) \int_{D} \frac{\partial u}{\partial t} L u d x \\
& +K^{2}(t) \int_{D} \sum_{i, j=1}^{n} \frac{\partial a_{i j}}{\partial t} \frac{\partial u}{\partial x_{i}} \frac{\partial u}{\partial x_{j}} d x
\end{aligned}
$$

Assume $u$ is a solution of

$$
\|L u(t)\|_{0} \leqq \phi(t)\|u(t)\|
$$

such that $u=0$ on $\Gamma \times I$, and let $\psi$ be a function satisfying

$$
\left|\int_{D} \sum_{i, j=1}^{n} \frac{\partial a_{i j}}{\partial t} \frac{\partial u}{\partial x_{i}} \frac{\partial u}{\partial x_{j}} d x\right| \leqq 2 \psi(t)\|u(t)\|^{2} .
$$

Applying Schwarz's inequality and (2.2) to the second term on the right-hand side of (2.1), and applying (2.3) to the third term of the same expression, we find that 


$$
\frac{d}{d t}\left[K^{2}(t)\|u(t)\|^{2}\right] \geqq 2 K^{2}(t)\|u(t)\|^{2}\left[\frac{K^{\prime}(t)}{K(t)}-f(t)\right],
$$

where we have set $f=\phi+\psi$. It follows that

$$
\frac{d}{d t} \log [K(t)\|u(t)\|] \geqq \frac{K^{\prime}(t)}{K(t)}-f(t)
$$

if $\|u(t)\| \neq 0$.

THEOREM. Let $u$ be a solution of (2.2) such that $u=0$ on $\Gamma \times I$. If $\left\|u\left(t_{0}\right)\right\| \neq 0$ and either

$$
\left(K^{\prime} / K\right)^{1 / p-1} f \in L_{p}(1, \infty) \text { for some } p, \quad 1 \leqq p<\infty,
$$

or

$$
K f / K^{\prime} \in L_{\infty}(1, \infty) \text { and }\left\|K f / K^{\prime}\right\|_{\infty} \leqq 1,
$$

then there exists a positive constant $C$ such that

$$
\|u(t)\| \geqq C\left\|u\left(t_{0}\right)\right\|[K(t)]^{-1}, \quad t \geqq t_{0} \geqq 1 .
$$

Proof. We first assume that $\|u(t)\| \neq 0$ for $t \geqq t_{0}$. Integrating (2.4) between $t_{0}$ and $t$ we obtain

$$
\log \frac{K(t)\|u(t)\|}{K\left(t_{0}\right)\left\|u\left(t_{0}\right)\right\|} \geqq \log \frac{K(t)}{K\left(t_{0}\right)}-\int_{t_{0}}^{t} f d s .
$$

In case (i), Hölder's inequality implies that

$$
\begin{aligned}
\left|\int_{t_{0}}^{t} f d s\right| & \leqq\left[\int_{t_{0}}^{t}\left|\left(\frac{K^{\prime}}{K}\right)^{-1 / q} f\right|^{p} d s\right]^{1 / p}\left[\int_{t_{0}}^{t} \frac{K^{\prime}}{K} d s\right]^{1 / q} \\
& \leqq N\left[\log \frac{K(t)}{K\left(t_{0}\right)}\right]^{1 / q},
\end{aligned}
$$

where $N$ is a constant and $1 / p+1 / q=1$. Hence, since $\lim _{t \rightarrow \infty} K(t)=\infty$, we see that the right-hand side of (2.6) is bounded below, and (2.5) follows. Under case (ii), the right-hand side of (2.6) is easily seen to be non-negative.

To prove that the assumption $\|u(t)\| \neq 0$ is valid for all $t \geqq t_{0}$, we suppose the contrary. Let $t_{1}>t_{0}$ be the least value of $t$ for which $\|u(t)\|$ $=0$. Then from the preceding result we find that (2.5) holds for $t_{0} \leqq t<t_{1}$. By the continuity of the norm, we must have $\left\|u\left(t_{1}\right)\right\| \neq 0$. This completes the proof of the theorem. 
If $K(t)=t^{\alpha}, \alpha>0$, conditions (i) and (ii) become: either $t^{1-1 / p} f$ $\in L_{p}(1, \infty)$ for some $p, 1 \leqq p<\infty$, or $t f \in L_{\infty}(1, \infty)$ and $\|t f\|_{\infty} \leqq \alpha$, which include Protter's conditions (1.3). For $K(t)=e^{\alpha t}, \alpha>0$, the conditions for the corresponding lower bound are: $f \in L_{p}(1, \infty)$ for some $p, 1 \leqq p$ $<\infty$, or $f \in L_{\infty}(1, \infty)$ and $\|f\|_{\infty} \leqq \alpha$. These include the conditions obtained by Protter in [5], and are comparable to those found by Protter [3], Cohen and Lees [2] and Agmon and Nirenberg [1] for solutions of parabolic inequalities.

3. Symmetric hyperbolic inequalities. Let $u$ be a $k$-component vector function in $C^{1}(R)$ and denote the components of $u$ by $u^{j}$, $j=1,2, \cdots, k$. For such functions we define

$$
L u=A_{0} \frac{\partial u}{\partial t}+\sum_{i=1}^{n} A_{i} \frac{\partial u}{\partial x_{i}}
$$

where the $A_{i}, i=0,1, \cdots, n$, are symmetric $k$-by- $k$ matrices with elements in $C^{1}(R)$, and $A_{0}$ is positive definite. We take as norms the quantities

$$
\begin{aligned}
& \|u(t)\|_{0}^{2}=\int_{D}(u, u) d x, \\
& \|u(t)\|^{2}=\int_{D}\left(A_{0} u, u\right) d x,
\end{aligned}
$$

with

$$
(u, v)=\sum_{j=1}^{k} u^{j} v^{j}
$$

Since $A_{0}$ is symmetric, we have

$$
\begin{aligned}
\frac{d}{d t}\|u(t)\|^{2} & =\int_{D}\left(2 A_{0} \frac{\partial u}{\partial t}+\frac{\partial A_{0}}{\partial t} u, u\right) d x \\
& =\int_{D}\left(2 L u-2 \sum_{i=1}^{n} A_{i} \frac{\partial u}{\partial x_{i}}+\frac{\partial A_{0}}{\partial t} u, u\right) d x
\end{aligned}
$$

Similarly, it follows that

$$
\int_{D}\left(A_{i} \frac{\partial u}{\partial x_{i}}, u\right) d x=-\frac{1}{2} \int_{D}\left(\frac{\partial A_{i}}{\partial x_{i}} u, u\right) d x
$$

for functions $u$ which vanish on the boundary $\Gamma \times I$. Thus, defining 


$$
B=\frac{\partial A_{0}}{\partial t}+\sum_{i=1}^{n} \frac{\partial A_{i}}{\partial x_{i}}
$$

we find that

$$
\frac{d}{d t}\|u(t)\|^{2}=\int_{D}(2 L u+B u, u) d x .
$$

Suppose $u$ is a solution of (2.2) and $u$ vanishes on $\Gamma \times I$. Let $\psi$ be a function satisfying

$$
\left|\int_{D}(B u, u) d x\right| \leqq 2 \psi(t)\|u(t)\|^{2} .
$$

Then the identity (3.1) implies that $u$ satisfies the inequality (2.4), so the theorem of $\S 2$ is also valid in the present case.

\section{BIBLIOGRAPHY}

1. S. Agmon and L. Nirenberg, Properties of solutions of ordinary differential equations in Banach space, Comm. Pure Appl. Math. 16 (1963), 121-239.

2. P. J. Cohen and M. Lees, Asymptotic decay of solutions of differential inequalities, Pacific J. Math. 11 (1961), 1235-1249.

3. M. H. Protter, Properties of solutions of parabolic equations and inequalities, Canad. J. Math. 13 (1961), 331-345.

4. - Asymptotic behavior and uniqueness theorems for hyperbolic equations and inequalities, Tech. Rep., Contract AF 49(638)-398, Univ. of Calif., Berkeley, Calif., 1960.

5. - Asymptotic behavior and uniqueness theorems for hyperbolic operators, Proc. of U.S.-U.S.S.R. Symposium on Partial Differential Equations, pp. 348-353, Novosibirsk, 1963.

University of California, Berkeley and University of California, Riverside 